\title{
Rayleigh Waves on an Exponentially Graded Poroelastic Half Space
}

\author{
Stan Chiriţă
}

Received: 29 August 2011 / Published online: 26 April 2012

(C) Springer Science+Business Media B.V. 2012

\begin{abstract}
In this paper we consider the propagation of seismic waves in isotropic poroelastic half spaces with continuously varying elastic properties, namely with an exponentially decaying depth profile. The present paper shows that the problem leads naturally to a bicubic equation. We obtain explicit inhomogeneous plane wave solutions in an exponential evanescent form with respect to the depth of half space. Further, these solutions are used to solve the boundary value problem of a Rayleigh surface wave and the secular equation is established. The results obtained theoretically are exemplified for numerical data and represented graphically for a representative poroelastic material.
\end{abstract}

Keywords Rayleigh waves - Elastic materials with voids · Exponentially graded half space $\cdot$ Secular equation

Mathematics Subject Classification 74J05 · 74J15 · 74M25

\section{Introduction}

In this paper we consider the theory of materials with voids introduced by Cowin and Nunziato [10]. The theory of elastic materials with voids is the simplest extension of the classical theory of elasticity. The basic idea of this theory is to suppose that there is a distribution of voids throughout the elastic body. Consequently, the bulk density is written as the product of two fields: the matrix material density field and the volume fraction field. This representation introduces an additional degree of kinematic freedom and it was employed previously by Goodman and Cowin [14] to develop a continuum theory of granular materials. The first investigations in the theory of thermoelastic materials with voids are due to Nunziato and

\footnotetext{
S. Chiriţă $(\bowtie)$

Faculty of Mathematics, Al. I. Cuza University of Iaşi, Blvd. Carol I, no. 11, 700506 Iaşi, Romania e-mail: schirita@uaic.ro

S. Chiriţă

Octav Mayer Institute of Mathematics, Romanian Academy, Bd. Carol I, no. 8, 700505 Iaşi, Romania
} 
Cowin [21] and Ieşan [16]. The Nunziato-Cowin theory has enjoyed much success in predicting various effects especially within the remit of linear theory, see, e.g., Ciarletta and Ieşan [6], Ieşan [17] and the recent work of Ghiba [12, 13] and Straughan [28] and the references therein. The intended applications of the theory concern the manufacture of building materials, e.g., plasterboard, brick, concrete.

On the other hand, wave propagation in an elastic material containing voids is an area with immense potential for practical applications. Many theories which describe the behaviour of materials with voids are developed and they proved to be useful in practice. In seismic zones buildings are constructed with much lighter porous materials and typically have thinner walls. As a consequence, there is a great need to study the acoustic properties of porous materials including the nature of the solid elastic matrix and the gas filling the pores, and the influence of temperature on these quantities. But seismology represents only one of the many fields where the theory of materials with voids is applied. Medicine, various branches of biology and oil exploration industry are other important fields of application.

We have to outline that the problem of homogeneous and acceleration waves propagation in materials with voids was studied in various works (see, e.g., Nunziato and Walsh [23, 24], Nunziato et al. [22], Cowin and Nunziato [10], Puri and Cowin [26], Chandrasekharaiah [1], Ciarletta and Straughan [7, 8], Ciarletta et al. [9], Chiriţă and Ghiba [2, 3] and a comprehensive review of the results can be found in the book by Straughan [28]. The use of acceleration waves and related analysis have proved extremely useful in recent investigations of wave motion in various dispersive and random media, in a variety of thermodynamic states, see, e.g., Ostoja-Starzewski and Trebicki [25], Puri and Jordan [27], Christov and Jordan [4], Jordan and Christov [19], Jordan and Puri [20] and Christov et al. [5].

Recently, Destrade [11] and Ting [29, 30] have used the Stroh formalism in order to study the wave propagation in the classical elasticity on an exponentially graded anisotropic half space.

In the present paper we consider the linear theory of materials with voids and then we study the seismic Rayleigh waves in a half space whose poroelastic properties are exponentially decaying away from the surface. Considering the direction of propagation and the normal to the surface both contained in the plane of symmetry $x_{1} O x_{2}$, the wave propagation results to in an appropriate two-dimensional wave and hence we are led to a state of plane strain problem based on a system of linear differential equations with variable coefficients. Using an idea devised first by Destrade [11], we transform this system in one with constant coefficients and then use the Stroh formulation to reduce it to a first-order differential system with constant coefficients. In this way we obtain inhomogeneous plane wave solutions in an exponential evanescent form with respect to the depth of half space. That means we arrive to a bicubic equation representing the propagation condition, whose coefficients are depending upon the decay rate of the poroelastic properties. Solving this equation we obtain explicit inhomogeneous plane wave solutions which are further used to find a solution for the boundary value problem of a Rayleigh surface wave. Thus, the secular equation for the wave speed is obtained. Furthermore, the reported results established theoretically are exemplified for numerical data and represented graphically for a representative poroelastic material. Finally, the results are applied for the boundary value problem of a Rayleigh surface wave in a homogeneous isotropic half space.

\section{Basic Equations}

Throughout this section $B$ is a bounded regular region of three-dimensional Euclidean space. We let $\bar{B}$ denote the closure of $B$, call $\partial B$ the boundary of $B$, and designate by $\mathbf{n}$ the outward 
unit normal of $\partial B$. We assume that the body occupying $B$ is a linearly elastic solid with voids. The body is referred to a fixed system of rectangular Cartesian axes $O x_{i}(i=1,2,3)$. Throughout this paper Latin indices have the range 1, 2, 3, Greek indices have the range 1,2 and the usual summation convention is employed. Moreover, a superposed dot denotes differentiation with respect to time and a subscript comma indicates partial differentiation with respect to the corresponding Cartesian coordinate.

Let $u_{i}$ be the components of the displacement vector field over $B$, and let $\varphi$ be the volume fraction field over $B$. The components of the linear strain measure are given by

$$
e_{i j}=\frac{1}{2}\left(u_{i, j}+u_{j, i}\right)
$$

and the constitutive equations for an inhomogeneous and isotropic elastic solid ${ }^{1}$ with voids are $[10,17]$

$$
\begin{aligned}
t_{i j} & =\lambda e_{r r} \delta_{i j}+2 \mu e_{i j}+\beta \varphi \delta_{i j}, \\
h_{i} & =\alpha \varphi_{, i}, \\
g & =-\beta e_{r r}-\xi \varphi .
\end{aligned}
$$

Here we have used the notation: $t_{i j}$ are the components of the stress tensor, $h_{i}$ are the components of the equilibrated stress vector, $g$ is the intrinsic equilibrated body force, $\delta_{i j}$ is Kronecker's delta and the constitutive coefficients $\lambda, \mu, \alpha, \beta$ and $\xi$ are prescribed smooth functions on $x$.

The equations of motion for the elastic material with voids are given by [10, 17]

$$
\begin{aligned}
& t_{j i, j}+\mathcal{F}_{i}=\varrho \ddot{u}_{i}, \\
& h_{i, i}+g+\mathcal{G}=\varrho \varkappa \ddot{\varphi},
\end{aligned}
$$

where $\varrho$ is the density mass depending on $x, \varkappa$ is the constant equilibrated inertia, $\mathcal{F}_{i}$ are the components of the body force vector per unit volume and $\mathcal{G}$ is the extrinsic equilibrated body force per unit volume. The surface force and the surface equilibrated force acting at a regular point $x$ on the surface $\partial B$ are given by

$$
\begin{aligned}
& t_{i}=t_{j i} n_{j}, \\
& h=h_{i} n_{i},
\end{aligned}
$$

where $n_{j}=\cos \left(\mathbf{n}_{x}, O x_{j}\right)$ and $\mathbf{n}_{x}$ is the unit vector of the outward normal to $\partial B$ at $x$.

The specific internal energy $W$ per unit volume for an isotropic elastic material with voids is defined by

$$
W=\mu e_{r s} e_{r s}+\frac{1}{2} \lambda e_{r r} e_{s s}+\frac{1}{2} \xi \varphi^{2}+\frac{1}{2} \alpha \varphi_{, r} \varphi_{, r}+\beta e_{r r} \varphi,
$$

and it is a positive definite quadratic form in terms of $e_{r s}, \varphi$ and $\varphi_{, n}$ if and only if

$$
\mu>0, \quad \alpha>0, \quad \xi>0, \quad\left(\lambda+\frac{2}{3} \mu\right) \xi>\beta^{2} .
$$

\footnotetext{
${ }^{1}$ Throughout this paper we will use the term isotropic in the sense defined by Gurtin [15], p. 71, that is the material at $\mathbf{x}$ is isotropic if the symmetry group at $\mathbf{x}$ equals the orthogonal group.
} 
The isotropic elastic material with voids is strongly elliptic if and only if

$$
\mu m_{r} m_{r} n_{s} n_{s}+\frac{1}{2} \lambda m_{r} m_{s} n_{r} n_{s}+\beta \eta m_{r} n_{r}+\frac{1}{2} \xi \eta^{2}+\frac{1}{2} \alpha \zeta_{r} \zeta_{r}>0
$$

for all real scalar $\eta$, unit vectors $\mathbf{m}=\left(m_{1}, m_{2}, m_{3}\right)$ and $\mathbf{n}=\left(n_{1}, n_{2}, n_{3}\right)$ and $\zeta=\left(\zeta_{1}, \zeta_{2}, \zeta_{3}\right)$. This is equivalent with the following inequalities (see, for example, $[2,3]$ )

$$
\mu>0, \quad \xi>0, \quad \alpha>0, \quad(\lambda+2 \mu) \xi>\beta^{2} .
$$

Throughout this paper we shall assume that the elastic material is strongly elliptic and hence (8) holds true.

\section{A State of Plane Strain in the Half Space with Exponential Depth Profile}

We assume that the region $B$ from here onwards refers to the half space $\Sigma: x_{2} \geq 0$ which is filled by an isotropic material with voids whose elastic properties are varying with the depth variable $x_{2}$. The half space is subject to no body loads. We consider the plane strain, parallel to the $x_{1} O x_{2}$-plane, characterized by

$$
\begin{aligned}
u_{\alpha} & =u_{\alpha}\left(x_{1}, x_{2}, t\right), \quad u_{3}=0, \\
\varphi & =\varphi\left(x_{1}, x_{2}, t\right), \quad\left(x_{1}, x_{2}\right) \in \Sigma, t \geq 0 .
\end{aligned}
$$

The above equation, in conjunction with the geometrical equation (1) and the constitutive equation (2) imply that $e_{i j}, t_{i j}, h_{i}$ and $g$ are all independent of $x_{3}$. It follows that the non-zero strain measures are given by

$$
e_{\alpha \beta}=\frac{1}{2}\left(u_{\alpha, \beta}+u_{\beta, \alpha}\right) .
$$

The constitutive equations show that the relevant non-zero dependent constitutive variables are $t_{\alpha \beta}, h_{\alpha}$ and $g$ and, moreover, we have

$$
\begin{gathered}
t_{\alpha \beta}=\lambda e_{\rho \rho} \delta_{\alpha \beta}+2 \mu e_{\alpha \beta}+\beta \varphi \delta_{\alpha \beta}, \\
h_{\rho}=\alpha \varphi_{, \rho}, \\
g=-\beta e_{\rho \rho}-\xi \varphi .
\end{gathered}
$$

According to our assumption that $\mathcal{F}_{i}=0, \mathcal{G}=0$, the equations of motion (3) reduce to

$$
\begin{aligned}
& t_{\beta \alpha, \beta}=\varrho \ddot{u}_{\alpha}, \\
& h_{\rho, \rho}+g=\varrho \varkappa \ddot{\varphi}, \quad \text { in } \Sigma \times(0, \infty) .
\end{aligned}
$$

We further suppose that the half space $x_{2} \geq 0$ is made of an isotropic elastic material with voids with an exponentially decaying depth profile, that is we assume that the elastic coefficients and the density mass are of the form

$$
\begin{array}{lll}
\lambda\left(x_{1}, x_{2}\right)=\lambda_{0} e^{-2 \sigma x_{2}}, & \mu\left(x_{1}, x_{2}\right)=\mu_{0} e^{-2 \sigma x_{2}}, & \varrho\left(x_{1}, x_{2}\right)=\varrho_{0} e^{-2 \sigma x_{2}}, \\
\alpha\left(x_{1}, x_{2}\right)=\alpha_{0} e^{-2 \sigma x_{2}}, & \beta\left(x_{1}, x_{2}\right)=\beta_{0} e^{-2 \sigma x_{2}}, & \xi\left(x_{1}, x_{2}\right)=\xi_{0} e^{-2 \sigma x_{2}},
\end{array}
$$


where $\lambda_{0}, \mu_{0}, \varrho_{0}, \alpha_{0}, \beta_{0}$ and $\xi_{0}$ are constants and $\sigma$ is a prescribed real positive number. We assume here that the equilibrated inertia $\varkappa$ is constant. We have to outline that at $\sigma=0$ the material is homogeneous. Such a case will be studied in detail in Sect. 7.

A substitution of (15) into basic equations (10)-(14) leads to a differential system with variable coefficients. This unpleasant feature can be avoided by using the following change of functions (see, for example, Destrade [11])

$$
u_{1}=U_{1}\left(x_{1}, x_{2}, t\right) e^{\sigma x_{2}}, \quad u_{2}=U_{2}\left(x_{1}, x_{2}, t\right) e^{\sigma x_{2}}, \quad \varphi=\phi\left(x_{1}, x_{2}, t\right) e^{\sigma x_{2}},
$$

and

$$
\begin{aligned}
& t_{11}=T_{11}\left(x_{1}, x_{2}, t\right) e^{-\sigma x_{2}}, \quad t_{22}=T_{22}\left(x_{1}, x_{2}, t\right) e^{-\sigma x_{2}}, \quad t_{12}=T_{12}\left(x_{1}, x_{2}, t\right) e^{-\sigma x_{2}}, \\
& h_{1}=H_{1}\left(x_{1}, x_{2}, t\right) e^{-\sigma x_{2}}, \quad h_{2}=H_{2}\left(x_{1}, x_{2}, t\right) e^{-\sigma x_{2}}, \quad g=G\left(x_{1}, x_{2}, t\right) e^{-\sigma x_{2}},
\end{aligned}
$$

so that the basic system (10)-(14) becomes

$$
\begin{gathered}
T_{11,1}+T_{12,2}=\sigma T_{12}+\varrho_{0} \ddot{U}_{1}, \\
T_{12,1}+T_{22,2}=\sigma T_{22}+\varrho_{0} \ddot{U}_{2}, \\
H_{1,1}+H_{2,2}+G=\sigma H_{2}+\varrho_{0} \varkappa \ddot{\phi}, \\
T_{11}=c_{11} U_{1,1}+c_{12}\left(U_{2,2}+\sigma U_{2}\right)+\beta_{0} \phi, \\
T_{22}=c_{12} U_{1,1}+c_{11}\left(U_{2,2}+\sigma U_{2}\right)+\beta_{0} \phi, \\
T_{12}=c_{66}\left(U_{1,2}+U_{2,1}+\sigma U_{1}\right), \\
H_{1}=\alpha_{0} \phi_{, 1}, \\
H_{2}=\alpha_{0}(\phi, 2+\sigma \phi), \\
G=-\beta_{0}\left(U_{1,1}+U_{2,2}+\sigma U_{2}\right)-\xi_{0} \phi,
\end{gathered}
$$

where

$$
c_{11}=\lambda_{0}+2 \mu_{0}, \quad c_{12}=\lambda_{0}, \quad c_{66}=\mu_{0} .
$$

It is worthy to observe that the differential system described by (18)-(20) leads to a secondorder differential system in terms of $\left\{U_{1}, U_{2}, \phi\right\}$ with constant coefficients.

In view of the assumptions described into (8), it follows that the constants $c_{11}, c_{12}, c_{66}$, $\varrho_{0}, \alpha_{0}, \beta_{0}$ and $\xi_{0}$ have to satisfy the following inequalities

$$
c_{66}>0, \quad \xi_{0}>0, \quad \alpha_{0}>0, \quad c_{11} \xi_{0}>\beta_{0}^{2} .
$$

\section{Inhomogeneous Plane Waves}

We consider the propagation of an inhomogeneous plane wave with speed $v$ and wave number $k$ in the $x_{1}$-direction in an inhomogeneous elastic half space $x_{2} \geq 0$ made of the isotropic 
elastic material with voids described in (15). Furthermore, we seek solutions of (18)-(20) in the form

$$
\left\{U_{1}, U_{2}, \phi\right\}\left(x_{1}, x_{2}, t\right)=\left\{v_{1}, v_{2}, \chi\right\}\left(x_{2}\right) e^{i k\left(x_{1}-v t\right)},
$$

and

$$
\left\{T_{11}, T_{22}, T_{12}, H_{1}, H_{2}, G\right\}\left(x_{1}, x_{2}, t\right)=i\left\{S_{11}, S_{22}, S_{12}, Q_{1}, Q_{2}, Q\right\}\left(x_{2}\right) e^{i k\left(x_{1}-v t\right)},
$$

in the half space $x_{2} \geq 0$ made of an isotropic elastic material with voids having an exponentially decaying profile as expressed in (15).

In view of the differential equations (18)-(20), we obtain for

$$
\mathcal{U}\left(x_{2}\right)=\left\{v_{1}, v_{2}, \chi, S_{12}, S_{22}, Q_{2}\right\}^{T}\left(x_{2}\right)
$$

the following first-order differential system

$$
\mathcal{U}^{\prime}\left(x_{2}\right)=i k \mathcal{A U}\left(x_{2}\right)
$$

where

$$
\begin{gathered}
\mathcal{A}=\left(\begin{array}{cc}
\mathcal{M} & \mathcal{N} \\
\mathcal{P} & \mathcal{Q}
\end{array}\right), \\
\mathcal{M}=\left(\begin{array}{ccc}
\frac{i \sigma}{k} & -1 & 0 \\
-\frac{c_{12}}{c_{11}} & \frac{i \sigma}{k} & \frac{i \beta_{0}}{k c_{11}} \\
0 & 0 & \frac{i \sigma}{k}
\end{array}\right), \quad \mathcal{N}=\left(\begin{array}{ccc}
\frac{1}{k c_{66}} & 0 & 0 \\
0 & \frac{1}{k c_{11}} & 0 \\
0 & 0 & \frac{1}{k \alpha_{0}}
\end{array}\right), \\
\mathcal{P}=\left(\begin{array}{c}
-k\left(c_{11}-\frac{c_{12}^{2}}{c_{11}}-\varrho_{0} v^{2}\right) \\
0 \\
-\beta_{0}\left(1-\frac{c_{12}}{c_{11}}\right) i \\
\varrho_{0} k v^{2}
\end{array} \quad \begin{array}{cc}
0 & -\alpha_{0} k-\frac{1}{k}\left(\xi_{0}-\frac{\beta_{0}^{2}}{c_{11}}\right)+\varrho_{0} \varkappa k v^{2}
\end{array}\right), \\
\mathcal{Q}=\left(\begin{array}{ccc}
-\frac{i \sigma}{k} & -\frac{c_{12}}{c_{11}} & 0 \\
-1 & -\frac{i \sigma}{k} & 0 \\
0 & -\frac{i \beta_{0}}{k c_{11}} & -\frac{i \sigma}{k}
\end{array}\right) .
\end{gathered}
$$

We remark that, in contrast to the case of a homogeneous material $(\sigma=0)$, the parameter dependence $\sigma$ enters in the differential system (26) in an explicit form by means of the matrices $\mathcal{M}$ and $\mathcal{Q}$.

\section{Solutions of the Transformed Differential System}

In this section we seek solutions of the differential system (26) in exponential evanescent form

$$
\mathcal{U}\left(x_{2}\right)=\mathcal{V} e^{i k r x_{2}},
$$

with

$$
\operatorname{Im}(r)>\frac{|\sigma|}{k},
$$


where $\mathcal{V}=\left\{\tilde{v}_{1}, \tilde{v}_{2}, \tilde{\chi}, \tilde{S}_{12}, \tilde{S}_{22}, \tilde{Q}_{2}\right\}^{T}$ is a constant vector, $r$ is a complex scalar, and the inequality $\operatorname{Im}(r)>|\sigma| / k$ ensures that

$$
\begin{aligned}
& \lim _{x_{2} \rightarrow \infty}\left\{u_{1}, u_{2}, \varphi\right\}\left(x_{1}, x_{2}, t\right)=0, \\
& \lim _{x_{2} \rightarrow \infty}\left\{t_{11}, t_{22}, t_{12}, h_{1}, h_{2}, g\right\}\left(x_{1}, x_{2}, t\right)=0, \quad \text { for all } x_{1} \in \mathbb{R}, t \geq 0,
\end{aligned}
$$

and

$$
\lim _{x_{2} \rightarrow \infty} \mathcal{U}\left(x_{2}\right)=0 .
$$

Concluding, we see that $\mathcal{V}$ and $r$ are solutions to the eigenvalue problem

$$
\mathcal{A} \mathcal{V}=r \mathcal{V}
$$

and hence the characteristic equation

$$
\operatorname{det}\left(r \mathcal{I}_{6}-\mathcal{A}\right)=0
$$

represents the propagation condition. Here $\mathcal{I}_{6}$ is the $6 \times 6$-unit matrix. Such an equation represents the vanishing of a $6 \times 6$ determinant and it can be written as

$$
p^{6}+P_{1} p^{4}+P_{2} p^{2}+P_{3}=0
$$

where

$$
\begin{gathered}
p^{2}=r^{2}+\frac{\sigma^{2}}{k^{2}}, \\
P_{1}=C_{1}+C_{2}+C_{3}, \\
P_{2}=C_{1} C_{2}+C_{2} C_{3}+C_{3} C_{1}-\frac{\varrho_{0} \beta_{0}^{2} v^{2}}{\alpha_{0} k^{2} c_{11}^{2}}+4 \frac{c_{12}}{c_{11}} \frac{\sigma^{2}}{k^{2}}, \\
P_{3}=C_{1} C_{2} C_{3}-\frac{\varrho_{0} \beta_{0}^{2} v^{2}}{\alpha_{0} k^{2} c_{11}^{2}} C_{2}-\frac{4 \sigma^{2}}{k^{2}}\left[\frac{\beta_{0}^{2}}{\alpha_{0} k^{2} c_{11}}\left(1-\frac{c_{12}}{c_{11}}\right)-\frac{c_{12}}{c_{11}} C_{3}\right], \\
C_{1}=1-\frac{\varrho_{0} v^{2}}{c_{11}}, \quad C_{2}=1-\frac{\varrho_{0} v^{2}}{c_{66}}, \\
C_{3}=1+\frac{\xi_{0} c_{11}-\beta_{0}^{2}}{\alpha_{0} k^{2} c_{11}}-\frac{\varrho_{0} \varkappa v^{2}}{\alpha_{0}} .
\end{gathered}
$$

The propagation condition (34) is a polynomial in $p$ with real coefficients and it represents the vanishing of a $6 \times 6$ determinant. When a homogeneous poroelastic half space is considered $(\sigma=0)$, the propagation condition in the form (35) can be explicitly solved as we will see in Sect. 7. In the case $\sigma \neq 0$, we can consider (35) as a cubic equation in $p^{2}$ and so it is clear that it has at least a real root which has to be non-positive in order to have $\operatorname{Im}(p)>0$. In fact, since the cubic equation (35) has all real coefficients, as a consequence of the intermediate value theorem, it follows that it has at least one solution $p^{2}$ among the real numbers. Moreover, if we set

$$
\Delta=18 P_{1} P_{2} P_{3}-4 P_{1}^{3} P_{3}+P_{1}^{2} P_{2}^{2}-4 P_{2}^{3}-27 P_{3}^{2},
$$


then we can distinguish several possible cases (see, for example, Irving [18], pp. 154-156)

- If $\Delta>0$, then the equation has three distinct real roots.

- If $\Delta=0$, then the equation has a multiple root and all its roots are real.

- If $\Delta<0$, then the equation has one real root and two nonreal complex conjugate roots.

In the first two cases the asymptotic condition (30) will be satisfied when

$$
\begin{aligned}
& P_{1}>0, \\
& P_{2}>0, \\
& P_{3}>0 .
\end{aligned}
$$

Let $p_{1}^{2}, p_{2}^{2}, p_{3}^{2}$ be the roots of the characteristic equation (35). Then, when the asymptotic condition (30) is taken into consideration, we can conclude that the only possibilities holding true are the following:

$$
p_{1}=i A, \quad p_{2}=i B, \quad p_{3}=i D,
$$

or

$$
p_{1}=i A, \quad p_{2}=E+i F, \quad p_{3}=-E+i F,
$$

where $A, B, D$ and $F$ are positive real numbers and $E$ is a real parameter. Therefore, we have

$$
\begin{aligned}
& p_{1}+p_{2}+p_{3}=i(A+B+D), \\
& p_{1} p_{2}+p_{2} p_{3}+p_{3} p_{1}=-(A B+B D+D A), \\
& p_{1} p_{2} p_{3}=-i A B D,
\end{aligned}
$$

or

$$
\begin{aligned}
& p_{1}+p_{2}+p_{3}=i(A+2 F), \\
& p_{1} p_{2}+p_{2} p_{3}+p_{3} p_{1}=-2 A F-E^{2}-F^{2}, \\
& p_{1} p_{2} p_{3}=-i A\left(E^{2}+F^{2}\right) .
\end{aligned}
$$

Anyway, half of all the roots of the propagation condition qualify as satisfying the decay condition (30). Let $r_{1}, r_{2}$ and $r_{3}$ be the three roots of characteristic equation (34) satisfying (30), corresponding to the roots $p_{1}^{2}, p_{2}^{2}, p_{3}^{2}$ of the characteristic equation (35).

Let us further introduce the notation

$$
p^{+}=r+\frac{i \sigma}{k}, \quad p^{-}=r-\frac{i \sigma}{k} .
$$

Then, the algebraic system (33) implies

$$
\begin{aligned}
& \tilde{S}_{12}=k c_{66} p^{-} \tilde{v}_{1}+k c_{66} \tilde{v}_{2}, \\
& \tilde{S}_{22}=k c_{12} \tilde{v}_{1}+k c_{11} p^{-} \tilde{v}_{2}-i \beta_{0} \tilde{\chi}, \\
& \tilde{Q}_{2}=k \alpha_{0} p^{-} \tilde{\chi},
\end{aligned}
$$


and

$$
\begin{aligned}
& \left(c_{66} p^{2}+c_{11}-\varrho_{0} v^{2}\right) \tilde{v}_{1}+\left(c_{66} p^{+}+c_{12} p^{-}\right) \tilde{v}_{2}-\frac{i \beta_{0}}{k} \tilde{\chi}=0, \\
& \left(c_{66} p^{-}+c_{12} p^{+}\right) \tilde{v}_{1}+\left(c_{11} p^{2}+c_{66}-\varrho_{0} v^{2}\right) \tilde{v}_{2}-\frac{i \beta_{0}}{k} p^{+} \tilde{\chi}=0 \\
& \frac{i \beta_{0}}{k} \tilde{v}_{1}+\frac{i \beta_{0}}{k} p^{-} \tilde{v}_{2}+\left(\alpha_{0} p^{2}+\alpha_{0}+\frac{\xi_{0}}{k^{2}}-\varrho_{0} \varkappa v^{2}\right) \tilde{\chi}=0
\end{aligned}
$$

In order that the algebraic system (47) to have a non-trivial solution it is necessary and sufficient that

$$
\left|\begin{array}{ccc}
c_{66} p^{2}+c_{11}-\varrho_{0} v^{2} & c_{66} p^{+}+c_{12} p^{-} & -\frac{i \beta_{0}}{k} \\
c_{66} p^{-}+c_{12} p^{+} & c_{11} p^{2}+c_{66}-\varrho_{0} v^{2} & -\frac{i \beta_{0}}{k} p^{+} \\
\frac{i \beta_{0}}{k} & \frac{i \beta_{0}}{k} p^{-} & \alpha_{0} p^{2}+\alpha_{0}+\frac{\xi_{0}}{k^{2}}-\varrho_{0} \varkappa v^{2}
\end{array}\right|=0
$$

Thus, the relation (48) represents the propagation condition expressed as vanishing a $3 \times 3$ determinant.

Furthermore, for each root $p_{s}$ of the propagation condition (35), we obtain the eigensolution $\mathcal{V}^{(s)}=\left\{\tilde{v}_{1}^{(s)}, \tilde{v}_{2}^{(s)}, \tilde{\chi}^{(s)}, \tilde{S}_{12}^{(s)}, \tilde{S}_{22}^{(s)}, \tilde{Q}_{2}^{(s)}\right\}^{T}$, with

$$
\begin{aligned}
& \tilde{v}_{1}^{(s)}=\frac{i \beta_{0}}{k c_{11}}\left[2 p_{s}^{2}-\left(p_{s}^{+}\right)^{2}+C_{2}\right], \\
& \tilde{v}_{2}^{(s)}=\frac{i \beta_{0}}{k c_{11}}\left[\left(p_{s}^{2}+1+C_{2}\right) p_{s}^{+}-p_{s}^{-}\right], \\
& \tilde{\chi}^{(s)}=p_{s}^{4}+\left(C_{1}+C_{2}\right) p_{s}^{2}+C_{1} C_{2}+4 \frac{c_{12}}{c_{11}} \frac{\sigma^{2}}{k^{2}},
\end{aligned}
$$

and

$$
\begin{aligned}
& \tilde{S}_{12}^{(s)}=\frac{i \beta_{0} c_{66}}{c_{11}}\left[\left(2 p_{s}^{2}+C_{2}-1\right) p_{s}^{-}+\left(C_{2}+1\right) p_{s}^{+}\right] \\
& \tilde{S}_{22}^{(s)}=\frac{i \beta_{0} c_{66}}{c_{11}}\left[-\left(1+C_{2}\right)\left(p_{s}^{2}+C_{2}\right)+\frac{2 i \sigma}{k}\left(p_{s}^{+}+p_{s}^{-}\right)+4 \frac{\sigma^{2}}{k^{2}}\right], \\
& \tilde{Q}_{2}^{(s)}=k \alpha_{0} p_{s}^{-}\left[p_{s}^{4}+\left(C_{1}+C_{2}\right) p_{s}^{2}+C_{1} C_{2}+4 \frac{c_{12}}{c_{11}} \frac{\sigma^{2}}{k^{2}}\right],
\end{aligned}
$$

for each $s=1,2,3$. We observe that the eigensolutions $\mathcal{V}^{(s)}, s=1,2,3$, depend on $\sigma$ in an explicit way as well as in an implicit manner by means of $p_{s}, p_{s}^{+}$and $p_{s}^{-}$.

\section{Seismic Rayleigh Waves}

In this section we consider the propagation of a seismic Rayleigh wave in an isotropic inhomogeneous Earth modelled as a poroelastic material. Thus, we consider that the half space $x_{2} \geq 0$ is made of the isotropic material with voids whose elastic properties are decaying with the depth variable $x_{2}$, as presented in (15). We assume that the body force and the equilibrated force are absent and the boundary surface $x_{2}=0$ is free of tractions and equilibrated 
tractions. Without loss of generality we will study the waves propagating along the $x_{1}$-axis and we will ignore an anti-plane deformation, that is we assume the following boundary surface conditions

$$
\begin{array}{ll}
t_{12}\left(x_{1}, 0, x_{3}, t\right)=0, & t_{22}\left(x_{1}, 0, x_{3}, t\right)=0, \\
h_{2}\left(x_{1}, 0, x_{3}, t\right)=0, & \text { for all } x_{1}, x_{3} \in \mathbb{R}, t \geq 0 .
\end{array}
$$

Moreover, we require that the solutions be attenuated in the direction $x_{2}$, so that they are decaying with distance from the plane surface $x_{2}=0$, that is we require that

$$
\begin{aligned}
& \lim _{x_{2} \rightarrow \infty}\left\{u_{1}, u_{2}, \varphi\right\}\left(x_{1}, x_{2}, x_{3}, t\right)=0, \\
& \lim _{x_{2} \rightarrow \infty}\left\{t_{11}, t_{22}, t_{12}, h_{1}, h_{2}, g\right\}\left(x_{1}, x_{2}, x_{3}, t\right)=0, \quad \text { for all } x_{1}, x_{3} \in \mathbb{R}, t \geq 0 .
\end{aligned}
$$

In what follows we will consider the boundary value problem of a Rayleigh surface wave, that is we will consider the boundary value problem defined by (10)-(14) and the boundary conditions (51) and the asymptotic decaying conditions (52). In view of the above analysis, we will treat the above problem by means of the inhomogeneous plane waves. Therefore, we seek solutions of the above half space problem in a linear combination of the three inhomogeneous plane waves, that is we take the general solution of the differential system (10)-(14) in the following form

$$
\begin{aligned}
u_{\varrho} & =\sum_{r=1}^{3} \gamma_{r} \tilde{v}_{\varrho}^{(r)} e^{i k\left(x_{1}-v t+p_{r}^{-} x_{2}\right)}, \\
\varphi & =\sum_{r=1}^{3} \gamma_{r} \tilde{\phi}^{(r)} e^{i k\left(x_{1}-v t+p_{r}^{-} x_{2}\right)}, \\
t_{\alpha \beta} & =i \sum_{r=1}^{3} \gamma_{r} \tilde{S}_{\alpha \beta}^{(r)} e^{i k\left(x_{1}-v t+p_{r}^{+} x_{2}\right)}, \\
h_{\varrho} & =i \sum_{r=1}^{3} \gamma_{r} \tilde{Q}_{\varrho}^{(r)} e^{i k\left(x_{1}-v t+p_{r}^{+} x_{2}\right)}, \\
g & =i \sum_{r=1}^{3} \gamma_{r} \tilde{G}^{(r)} e^{i k\left(x_{1}-v t+p_{r}^{+} x_{2}\right)},
\end{aligned}
$$

where $\gamma_{r}, r=1,2,3$, are parameters at our disposal to be determined. Such functions satisfy the basic equations (10)-(14) and the asymptotic decay conditions (52). While the boundary conditions (51) furnish the following algebraic system for determining the parameters $\gamma_{r}$, $r=1,2,3$,

$$
\begin{aligned}
\gamma_{1} \tilde{S}_{12}^{(1)}+\gamma_{2} \tilde{S}_{12}^{(2)}+\gamma_{3} \tilde{S}_{12}^{(3)} & =0, \\
\gamma_{1} \tilde{S}_{22}^{(1)}+\gamma_{2} \tilde{S}_{22}^{(2)}+\gamma_{3} \tilde{S}_{22}^{(3)} & =0, \\
\gamma_{1} \tilde{Q}_{2}^{(1)}+\gamma_{2} \tilde{Q}_{2}^{(2)}+\gamma_{3} \tilde{Q}_{2}^{(3)} & =0 .
\end{aligned}
$$


Table 1 Surface wave speed

\begin{tabular}{llrl}
\hline$\sigma$ & $v$ & $\sigma$ & $v$ \\
\hline 0 & 0.0561330177 & & \\
1 & 0.0368950008 & 6 & 0.0102224018 \\
2 & 0.0253317513 & 7 & 0.0088378461 \\
3 & 0.0187885540 & 8 & 0.0077774255 \\
4 & 0.0147647128 & 9 & 0.0069408084 \\
5 & 0.0120987117 & 10 & 0.0062647070 \\
\hline
\end{tabular}

In order that the system (55) to have a non-trivial solution it is necessary and sufficient that

$$
\left|\begin{array}{ccc}
\tilde{S}_{12}^{(1)} & \tilde{S}_{12}^{(2)} & \tilde{S}_{12}^{(3)} \\
\tilde{S}_{22}^{(1)} & \tilde{S}_{22}^{(2)} & \tilde{S}_{22}^{(3)} \\
\tilde{Q}_{2}^{(1)} & \tilde{Q}_{2}^{(2)} & \tilde{Q}_{2}^{(3)}
\end{array}\right|=0
$$

In view of the relation (50), (56) represents the secular equation for the wave speed $v$ in the case of the traction free and equilibrated traction free half space $x_{2}=0$. It should be pointed out that a secular equation can be easily established when the Earth surface is free of traction and it is maintained at zero volume fraction.

In view of relation (50), from (56) we can get an idea on how the secular equation depends on the decay/growth rate $\sigma$ of the poroelastic profile. Obviously, when $\sigma=0$, (56) will give the secular equation for the wave speed in a homogeneous poroelastic half space. An explicit discussion in this case will be given in Sect. 7.

Relating to the inhomogeneous poroelastic half space, in Table 1 we give the solution of the secular equation for a representative isotropic (fictitious) material with voids as considered by Puri and Cowin [26], for a unit wave number and various values of decay/growth rate of depth profile. That means we consider a material with voids with the following properties: $\lambda_{0}=15 \mathrm{GPa}, \mu_{0}=7.5 \mathrm{GPa}, \xi_{0}=12 \mathrm{GPa}, \beta_{0}=10 \mathrm{GPa}, \alpha_{0}=8 \mathrm{GPa}, \varrho_{0}=2000 \mathrm{~kg} / \mathrm{m}^{3}$, $\varkappa=1.6 \mathrm{~m}^{2}$ and we take the wave number $k=1 \mathrm{~m}^{-1}$ and the parameter $\sigma$ ranges in the set $\{0,1,2, \ldots, 10\}$. We can observe a rapid decay of the wave speed $v$ from the value $v_{0}=0.0561330177$ (corresponding to the homogeneous material) with respect to the growth of the parameter $\sigma$ from 0 to 10 .

\section{Rayleigh Waves for Isotropic Homogeneous Poroelastic Materials}

In the case of isotropic homogeneous poroelastic materials we have $\sigma=0$, and hence $p=r$, and therefore, the propagation condition (35) takes the simplified form

$$
\left(r^{2}+C_{2}\right)\left[r^{4}+\left(C_{1}+C_{3}\right) r^{2}+C_{1} C_{3}-\frac{\varrho_{0} \beta_{0}^{2} v^{2}}{\alpha_{0} k^{2} c_{11}^{2}}\right]=0 .
$$

Thus, we have

$$
p_{3}^{2}=r_{3}^{2}=-C_{2},
$$

while the other two eigenvalues $p_{1}^{2}=r_{1}^{2}, p_{2}^{2}=r_{2}^{2}$ satisfy the equation

$$
r^{4}+\left(C_{1}+C_{3}\right) r^{2}+C_{1} C_{3}-\frac{\varrho_{0} \beta_{0}^{2} v^{2}}{\alpha_{0} k^{2} c_{11}^{2}}=0
$$


and hence

$$
r_{1,2}^{2}=\frac{1}{2}\left[-C_{1}-C_{3} \pm \sqrt{\left(C_{1}-C_{3}\right)^{2}+\frac{4 \varrho_{0} \beta_{0}^{2} v^{2}}{\alpha_{0} k^{2} c_{11}^{2}}}\right] .
$$

The above quantities are admissible roots if and only if the inequality

$$
\operatorname{Im}\left(r_{s}\right)>0,
$$

holds true. So, we must have

$$
C_{2}>0, \quad C_{1}+C_{3}>0, \quad C_{1} C_{3}-\frac{\varrho_{0} \beta_{0}^{2} v^{2}}{\alpha_{0} k^{2} c_{11}^{2}}>0,
$$

that is

$$
C_{1}>0, \quad C_{2}>0, \quad C_{3}>0, \quad C_{1} C_{3}>\frac{\varrho_{0} \beta_{0}^{2} v^{2}}{\alpha_{0} k^{2} c_{11}^{2}} .
$$

Further, these inequalities are satisfied if and only if the wave speed $v$ is so that

$$
v^{2}<\min \left\{\mathfrak{c}_{1}, \mathfrak{c}_{2}, \mathfrak{c}_{3}, \mathfrak{c}_{4}\right\},
$$

or

$$
v^{2}<\min \left\{\mathfrak{c}_{1}, \mathfrak{c}_{2}, \mathfrak{c}_{3}\right\} \text { and } v^{2}>\mathfrak{c}_{5},
$$

where

$$
\begin{gathered}
\mathfrak{c}_{1}=\frac{c_{11}}{\varrho_{0}}, \\
\mathfrak{c}_{2}=\frac{c_{66}}{\varrho_{0}}, \\
\mathfrak{c}_{3}=\frac{\alpha_{0} c_{11} k^{2}+c_{11} \xi_{0}-\beta_{0}^{2}}{\varrho_{0} \varkappa k^{2} c_{11}}, \\
\mathfrak{c}_{4}=\frac{c_{11}}{2 \varrho_{0}}\left\{1+\frac{\alpha_{0} k^{2}+\xi_{0}}{\varkappa c_{11} k^{2}}-\sqrt{\left(1-\frac{\alpha_{0} k^{2}+\xi_{0}}{\varkappa c_{11} k^{2}}\right)^{2}+\frac{4 \beta_{0}^{2}}{c_{11}^{2} k^{2} \varkappa}}\right\}>0, \\
\mathfrak{c}_{5}=\frac{c_{11}}{2 \varrho_{0}}\left\{1+\frac{\alpha_{0} k^{2}+\xi_{0}}{\varkappa c_{11} k^{2}}+\sqrt{\left(1-\frac{\alpha_{0} k^{2}+\xi_{0}}{\varkappa c_{11} k^{2}}\right)^{2}+\frac{4 \beta_{0}^{2}}{c_{11}^{2} k^{2} \varkappa}}\right\} .
\end{gathered}
$$

It can be easily seen that

$$
\mathfrak{c}_{4}<\mathfrak{c}_{3}<\mathfrak{c}_{5},
$$

and hence the relation (65) cannot be true. On the other hand, we have

$$
\mathfrak{c}_{1}>\mathfrak{c}_{4},
$$

and therefore, the roots $r_{s}, s=1,2,3$, given by (58) and (60), are admissible if and only if

$$
v^{2}<\min \left(\mathfrak{c}_{2}, \mathfrak{c}_{4}\right) .
$$


Furthermore, for each root $p_{s}=r_{s}, s=1,2,3$, we obtain the eigensolution $\mathcal{V}^{(s)}=$ $\left\{\tilde{v}_{1}^{(s)}, \tilde{v}_{2}^{(s)}, \tilde{\chi}^{(s)}, \tilde{S}_{12}^{(s)}, \tilde{S}_{22}^{(s)}, \tilde{Q}_{2}^{(s)}\right\}^{T}$, with

$$
\begin{aligned}
\tilde{v}_{1}^{(\alpha)} & =\frac{i \beta_{0}}{k c_{11}} \\
\tilde{v}_{2}^{(\alpha)} & =\frac{i \beta_{0}}{k c_{11}} r_{\alpha} \\
\tilde{\chi}^{(\alpha)} & =r_{\alpha}^{2}+C_{1}, \quad \alpha=1,2,
\end{aligned}
$$

and

$$
\begin{aligned}
\tilde{S}_{12}^{(\alpha)} & =\frac{2 i \beta_{0} c_{66}}{c_{11}} r_{\alpha} \\
\tilde{S}_{22}^{(\alpha)} & =-\frac{i \beta_{0} c_{66}}{c_{11}}\left(1+C_{2}\right), \\
\tilde{Q}_{2}^{(\alpha)} & =k \alpha_{0} r_{\alpha}\left(r_{\alpha}^{2}+C_{1}\right), \quad \alpha=1,2,
\end{aligned}
$$

while

$$
\begin{aligned}
& \tilde{v}_{1}^{(3)}=-r_{3}, \\
& \tilde{v}_{2}^{(3)}=1, \\
& \tilde{\chi}^{(3)}=0,
\end{aligned}
$$

and

$$
\begin{aligned}
& \tilde{S}_{12}^{(3)}=k c_{66}\left(1+C_{2}\right), \\
& \tilde{S}_{22}^{(3)}=2 k c_{66} r_{3}, \\
& \tilde{Q}_{2}^{(3)}=0 .
\end{aligned}
$$

Therefore, the secular equation (56) becomes

$$
\left|\begin{array}{ccc}
\frac{2 i \beta_{0} c_{66}}{c_{11}} r_{1} & \frac{2 i \beta_{0} c_{66}}{c_{11}} r_{2} & k c_{66}\left(1+C_{2}\right) \\
-\frac{i \beta_{0} c_{66}}{c_{11}}\left(1+C_{2}\right) & -\frac{i \beta_{0} c_{66}}{c_{11}}\left(1+C_{2}\right) & 2 k c_{66} r_{3} \\
k \alpha_{0} r_{1}\left(r_{1}^{2}+C_{1}\right) & k \alpha_{0} r_{2}\left(r_{2}^{2}+C_{1}\right) & 0
\end{array}\right|=0
$$

that is

$$
\left(r_{2}-r_{1}\right)\left[4 r_{1} r_{2} r_{3}\left(r_{1}+r_{2}\right)+\left(1+C_{2}\right)^{2}\left(r_{1}^{1}+r_{2}^{2}+r_{1} r_{2}+C_{1}\right)\right]=0 .
$$

Assuming that the three roots are distinct, it follows that the secular equation is

$$
4 r_{1} r_{2} r_{3}\left(r_{1}+r_{2}\right)+\left(1+C_{2}\right)^{2}\left(r_{1} r_{2}-C_{3}\right)=0
$$


Fig. 1 The graphic of the secular function $S(v)$

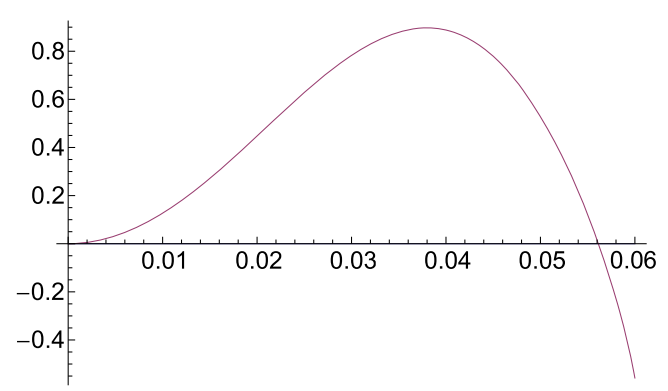

In view of relations (58)-(61), we have

$$
\begin{aligned}
r_{1} r_{2} & =-\sqrt{C_{1} C_{3}-\frac{\varrho_{0} \beta_{0}^{2} v^{2}}{\alpha_{0} k^{2} c_{11}^{2}}}, \\
r_{1}+r_{2} & =i \sqrt{C_{1}+C_{3}+2 \sqrt{C_{1} C_{3}-\frac{\varrho_{0} \beta_{0}^{2} v^{2}}{\alpha_{0} k^{2} c_{11}^{2}}}},
\end{aligned}
$$

and hence the secular equation (77) becomes

$$
\begin{aligned}
S(v) \equiv & 4 \sqrt{C_{1} C_{2} C_{3}-\frac{\varrho_{0} \beta_{0}^{2} v^{2}}{\alpha_{0} k^{2} c_{11}^{2}} C_{2}} \sqrt{C_{1}+C_{3}+2 \sqrt{C_{1} C_{3}-\frac{\varrho_{0} \beta_{0}^{2} v^{2}}{\alpha_{0} k^{2} c_{11}^{2}}}}- \\
& -\left(1+C_{2}\right)^{2}\left(C_{3}+\sqrt{C_{1} C_{3}-\frac{\varrho_{0} \beta_{0}^{2} v^{2}}{\alpha_{0} k^{2} c_{11}^{2}}}\right)=0 .
\end{aligned}
$$

Such an equation was discussed in [3] and it was shown there that it has at least a solution.

For the representative isotropic (fictitious) material with voids considered by Puri and Cowin [26], with $\sigma=0$, the variation of the function $S(v)$ is described by the graphic expressed in the Fig. 1. It becomes clear from this picture that the secular equation has an admissible solution.

Acknowledgements The author is grateful to the reviewer for his useful comments. The author gratefully acknowledges the financial support of the Romanian Ministry of Education and Research and Innovation through the CNCS grant code ID-89, Contract No. 457.

\section{References}

1. Chandrasekharaiah, D.S.: Surface waves in an elastic half-space with voids. Acta Mech. 62, 77-85 (1986)

2. Chiriţă, S., Ghiba, I.D.: Strong ellipticity and progressive waves in elastic materials with voids. Proc. R. Soc. A, Math. Phys. Eng. Sci. 466, 439-458 (2010)

3. Chiriță, S., Ghiba, I.D.: Inhomogeneous plane waves in elastic materials with voids. Wave Motion 47, 333-342 (2010)

4. Christov, C.I., Jordan, P.M.: Heat conduction paradox involving second sound propagation in moving media. Phys. Rev. Lett. 94, 154301 (2005)

5. Christov, I., Jordan, P.M., Christov, C.I.: Nonlinear acoustic propagation in homentropic perfect gases: a numerical study. Phys. Lett. A 353, 273-280 (2006) 
6. Ciarletta, M., Ieşan, D.: Non-Classical Elastic Solids. Longman Scientific and Technical, Wiley, Harlow, New York (1993)

7. Ciarletta, M., Straughan, B.: Poroacoustic acceleration waves. Proc. R. Soc. Lond. Ser. A, Math. Phys. Sci. 462, 3493-3499 (2006)

8. Ciarletta, M., Straughan, B.: Poroacoustic acceleration waves with second sound. J. Sound Vib. 306, 725-731 (2007)

9. Ciarletta, M., Straughan, B., Zampoli, V.: Thermo-poroacoustic acceleration waves in elastic materials with voids without energy dissipation. Int. J. Eng. Sci. 45, 736-743 (2007)

10. Cowin, S.C., Nunziato, J.W.: Linear elastic materials with voids. J. Elast. 13, 125-147 (1983)

11. Destrade, M.: Seismic Rayleigh waves on an exponentially graded, orthotropic half-space. Proc. R. Soc. A, Math. Phys. Eng. Sci. 463, 495-502 (2007)

12. Ghiba, I.D.: Semi-inverse solution for Saint-Venant's problem in the theory of porous elastic materials. Eur. J. Mech. A, Solids 27, 1060-1074 (2008)

13. Ghiba, I.D.: Spatial estimates concerning the harmonic vibrations in rectangular plates with voids. Arch. Mech. 60, 263-279 (2008)

14. Goodman, M.A., Cowin, S.C.: A continuum theory for granular materials. Arch. Ration. Mech. Anal. 44, 249-266 (1972)

15. Gurtin, M.E.: The linear theory of elasticity. In: Truesdell, C. (ed.) Flügge's Handbuch der Physik, vol. VIa/2, pp. 1-295. Springer, Berlin-Heidelberg-New York (1972)

16. Ieşan, D.: A theory of thermoelastic materials with voids. Acta Mech. 60, 67-89 (1986)

17. Ieşan, D.: Thermoelastic Models of Continua. Kluwer Academic, London (2004)

18. Irving, R.S.: Integers, Polynomials, and Rings. Springer, New York (2004)

19. Jordan, P.M., Christov, C.I.: A simple finite difference scheme for modelling the finite-time blow-up of acoustic acceleration waves. J. Sound Vib. 281, 1207-1216 (2005)

20. Jordan, P.M., Puri, P.: Growth/decay of transverse acceleration waves in nonlinear elastic media. Phys. Lett. A 341, 427-434 (2005)

21. Nunziato, J.W., Cowin, S.C.: A nonlinear theory of elastic materials with voids. Arch. Ration. Mech. Anal. 72, 175-201 (1979)

22. Nunziato, J.W., Kennedy, J.E., Walsh, E.K.: The behavior of one-dimensional acceleration waves in an inhomogeneous granular solid. Int. J. Eng. Sci. 16, 637-648 (1978)

23. Nunziato, J.W., Walsh, E.K.: On the influence of void compaction and material non-uniformity on the propagation of one-dimensional acceleration waves in granular materials. Arch. Ration. Mech. Anal. 64, 299-316 (1977)

24. Nunziato, J.W., Walsh, E.K.: Addendum "On the influence of void compaction and material nonuniformity on the propagation of one-dimensional acceleration waves in granular materials". Arch. Ration. Mech. Anal. 67, 395-398 (1978)

25. Ostoja-Starzewski, M., Trebicki, J.: On the growth and decay of acceleration waves in random media. Proc. R. Soc. Lond. Ser. A, Math. Phys. Sci. 455, 2577-2614 (1999)

26. Puri, P., Cowin, S.C.: Plane waves in linear elastic materials with voids. J. Elast. 15, 167-183 (1985)

27. Puri, P., Jordan, P.M.: On the propagation of plane waves in type-III thermoelastic media. Proc. R. Soc. Lond. Ser. A, Math. Phys. Sci. 460, 3203-3221 (2004)

28. Straughan, B.: Stability and Wave Motion in Porous Media. Springer, New York (2008)

29. Ting, T.C.T.: Secular equations for Rayleigh and Stoneley waves in exponentially graded elastic materials of general anisotropy under the influence of gravity. J. Elast. 105, 331-347 (2011)

30. Ting, T.C.T.: Surface waves in an exponentially graded, general anisotropic elastic material under the influence of gravity. Wave Motion 48, 335-344 (2011) 